\title{
SOLITON/EXCITON TRANSPORT IN PROTEINS
}

\author{
ZACHARIAH SINKALA*
}

\begin{abstract}
.
The study of electron/proton transport in $\alpha$-helix sections of proteins have illustrated the existence of soliton-like mechanisms. This paper investigates the existence possible like soliton-type mechanisms in other parts of the protein. We use classical Hamiltonian analysis in our investigations as opposed to Quantum Hamiltonian analysis which was used by Ciblis and Cosic in studying the same problem.
\end{abstract}

Key words. Soliton, transport in proteins, Classical Hamiltonian, Klein Gordon.

AMS(MOS) subject classifications. Primary 92C30, 81Q05.

1. Introduction. Many biological processes are associated with a space propagation of energy and electrons along protein molecules. For example, the energy released (under normal physiological conditions releases $0.42 \mathrm{eV}$ of energy) under hydrolysis of ATP molecule. The major question is What happens to this energy? How does it perform useful work? Is the energy used through non-equilibrium process or does it thermalize and then work through an equilibrium processes? One hypothesis is some cases is trasferred along $\alpha$-helical protein molecules as the vibration oscillation of atoms $C=O$ of peptide groups contained in these molecules. This energy is about half $\left(.21 \mathrm{eV}\right.$ or $\left.1665 \mathrm{~cm}^{-1}\right)$ of the energy released during ATP hydrolysis. Moreover, the amide-I vibration stays nearly constant from protein to protein, indicating that it is rather weakly coupled to other degrees of freedom. All these factors lead to the assumption that energy released by ATP might stay localised and stored in the amide-I vibration, for example see Davydov [13]. He suggested that the amide-I energy could stay localised through nonlinear interactions of the vibrational excitation and the deformation in the protein structure caused by the presence of the excitation. The excitation and the deformation balance each other and form a soliton. Therefore, a soliton is a localized packet of energy. Protein molecules also transport electrons from donors to acceptors very effectively.

There is also much evidence that shows that biological processes can be induced or modualted by the induction of light of particular frequencies, for example see the work Cosics[8] and [10]. This is caused directly by light-induced changes in the energitic states of molecules and in particular proteins. The function of some proteins (proton pumps) is directly connected with absorption of visible light of defined wavelengths as in the case of rhodopsins. The strong light absorption is due to the presence of a color

*Department of Mathematical Sciences, Middle Tennessee State University, Murfreesboro, Tennessee 37132. This work was supported in part by NIA grant from Middle Tennessee State University. 
prosthetic group bound to the protein, whilist the frequency selectivity of this absorption is defined by the amino acid sequences of the protein[8],[10].

On the other hand, there is evidence that light of defined frequency can induce or enhance some biological processes which are normally controlled only by proteins,see Cosic[8]. All these frequency selective effects of light on biological processes involving protein activation involves ernergies of the same order and nature as electromagnetic irradiation of light. These phenomena are discussed in terms of the Resonant recognition Model(RRM) which proposes that the protein interactions are based on resonant electromagnetic energy transfer within the range of infra-red and visible light, see Cosic[8].

2. A summarized discussion of RRM model. All protein molecul-es are made from a linear sequence of amino acids. The RRM model interprets this linear information using signal analysis methods [8]. Firstly, the amino acid sequences are transformed into numerical series using the electron-ion interaction potential for each amino acid

(Veljkovic and Slavic[40]). Their values describe the average states of valence electrons in the amino acid. Numerical series obtained in this way can then be transformed into the frequency domain using Fast Fourier Tranform in order to extract information pertinent to the biological function.

To determine the common frequency components for a group of protein sequences, the absolute values of multiples cross-spectral function coefficients are calculated. Peak frequencies in the multiple cross-spectral function denote common frequency components for the analysed sequences. Signal to noise ratio for each peak was considered as measure of similarity between analysed sequences. This ratio was calculated as the ratio between signal intensity at the particular peak frequency and the spectrum mean value(ratio of least 20 sequence is considered significant). The presence of a peak with significant ratio in a multiple cross spectral function of a group of sequences with the same biological function means that all of the analysed sequences within the group have this frequency component in common. This frequency is related to the biological function as it was found in previous investigations [8] that:

(i) such a peak exists only for the group of proteins with the same biological function;

(ii) no significant peak exists for biologically unrelated proteins; and

(iii) peak frequencies are different for different biological functions.

Furthermore, it was shown that the proteins and their targets have the same characteristic frequency in common, see Cosic [8],[10]. Therefore, it can be concluded that these frequencies characterized not only general function but also recognition and interaction between particular protein and its target. This interaction can be considered as resonant energy tranfer between interacting molecules. This energy can be transfered through oscillations of a physical field possibly electromagnetic in its nature. As 
there is evidence that proteins have certain conducting or semiconducting properties(see Davydov[13])then charge moving through the protein backbone and passing different energy levels caused by different amino acid side groups can produce sufficient conditions for the specific electromagnetic radiation or absorbtion. The frequency range of this field depends on charge velocity estimated to be $7.87 \times 10^{5} \mathrm{~ms}^{-1}$ and the distance between amino acids in protein which is $3.8 \AA$, see Cosic[8],[10]. Having this in mind, the frequency range obtained for protein interactions was $10^{13}$ to $10^{15} \mathrm{~Hz}$, see Cosic[8],[10]. All the results obtained in RRM model lead to the the conclusion that specificity of protein interactions are based on the resonant electromagnetic energy transfer on a frequency specific for each observed interaction. This has been tested on a number of examples including light obsorbing proteins (Cosic[8]), growth factor activation(Cosic[6],[7]), enzyme activation(Cosic[9]) and red/far red and blue light receptors in plants(Cosic and Birch[11]). The physical basis of RRM postulates is the possibility of charge tranfer through protein backbone. The possibility and nature of charge or energy transfer along the protein backbone is discussed here.

3. The problem under investigation. This paper investigates the possiblity of a soliton-like mechanim being involved in the resonance recognition process. As the RRM derives a set of frequencies computed from data on the whole length of the protein, it has been assumed that the charge or excitations travelling along the back bone can produce vibrations(oscillations) of particular frequencies. The assumptions here is that solitons would travel along the backbone. Therefore, the quotient of soliton velocity/length of protein backbone could be of the order of RRM frequencies.

4. Preliminary work and known results on soliton-like mechanisms in proteins. There are instances of protein to protein reactions where an electron is transfered 30-70 from the reactive site unassisted by a chemical carrier. The energy required for an electron to escape its ground state in a protein is approximately $2.3-3.5 \mathrm{eV}$. The background thermal energy at $300^{\circ} \mathrm{K}$ is approximately $0.025 \mathrm{eV}$ and optical excitation of the protein is unlikely,see Kharkyanen [23]. The electron transfer is difficult to explain with either standard chemical theory or by quantum mechanical tunneling,see, Davydov[13].

One explanation to the problem of electron transport involves the introduction of solitons. The general properties of solitons are solitons are solitary waves(waves localized in space) with the following properties:

1. they preserves their shape and velocities.

2. they are extremely stable to perturbations(In particular collisions with small amplitude linear waves),

3. they are even stable with respect to collusions with other solitons. In such collusion they pass through each other and recover their speed and shape after interaction. The outcome of the collusion of two solitons is a 
simple phase shift of each excitation.

Davydov[13] investigated the conditions that would necessitate the formation of excitons and solitons within proteins. His simplest model considers only resonant interactions of vibrational excitations of peptide groups and this was extended to cover the three spine model for the $\alpha$-protein. His model assumes that there there is a dipole-dipole interaction between the blocks and that there is perturbation of the bond structure within the blocks. This model is equally applicable to electron transport. Careful inspection of the $\alpha$-helix structure of proteins reveals three channels situated approximately in the longitudal direction of the sequence

$$
\begin{gathered}
H-N-C=O \ldots H-N-C=O \ldots H-N-C=O \ldots H-N-C=O \ldots \\
\ldots H-N-C=O \ldots H-N-C=O
\end{gathered}
$$

where the dotted lines represent hydrogen bonds. For detailed analysis it is necessary to consider the interaction of all three channels. He(Davydov) first considered a one-dimensional periodic array of block of atoms:

Here we will only consider one, since it suffices to convey the basic idea. The Hamiltonnian Davydov used to describe the situation is:

$$
\begin{gathered}
H=\sum_{n}\left[E_{0} B_{n}^{\dagger} B_{n}-J\left(B_{n+1}^{\dagger} B_{n}+B_{n}^{\dagger} B_{n+1}\right)\right] \\
+\sum_{n}\left[\frac{v_{n}}{2 m}+\frac{1}{2} w\left(u_{n+1}-u_{n}\right)^{2}\right]+\chi \sum_{n}\left(u_{n+1}-u_{n-1}\right) B_{n}^{\dagger} B_{n} \\
=\hat{H}_{C O}+\hat{H}_{p h}+\hat{H}_{i n t} .
\end{gathered}
$$

Here, $B_{n}^{\dagger}$ and $B_{n}$ are boson creation annihilation operators for quanta of intramolecular vibrations with energy $E_{0}=1665 \mathrm{~cm}^{-1}$ at site n ( the $C O$ stretch mode or amide-I mode), $u_{n}$ and $v_{n}$ are the molecular displacement and momentum operators for the molecule at site $\mathrm{n}$ (the entire peptide group), $\mathrm{m}$ and $\mathrm{w}$ are the molecular mass and intermolecular force constant, and $\mathrm{J}$ is the intersite transfer energy produced by dipole-dipole interactions. The nonlinear coupling constant $\chi$ arises from the modulation of the onsite by the molecular displacements. It is the derivative of the amide-I energy with respect to the length between peptide groups(l) of the adjacent hydrogen bond:

$$
\chi \equiv \frac{d E_{0}}{d l}
$$


The vibration part $\hat{H}_{C O}$, the phonon part $\hat{H}_{p h}$, and the interaction part $\hat{H}_{i n t}$ are defined to be individual terms in (4.1).

For later comparison we write here the equation of motion for the Heisenberg operator $B_{n}(t)$,

$$
i \hbar \dot{B}_{n}=E_{0} B_{n}-J\left(B_{n+1}+B_{n-1}\right)+\chi B_{n}\left(u_{n+1}-u_{n-1}\right) .
$$

The form of this equation is such that a phase transformation

$$
B_{n}(t)=\tilde{B}_{n}(t) \exp \left(\frac{-i E_{0} t}{\hbar}\right)
$$

removes the energy of the amide-I quantum from the equation, that is, the eqution for $\tilde{B}_{n}(t)$ is $(4.2)$ but without the term proportional to $E_{0}$.

Davydov minimizes the average value of $H$ with respect to some wave function. This leads to the differential-difference equations. Extensive numerical and theoretical analysis of these differential-difference equations yields the following results: It is reasonable to expect soliton formation at the level of energy released by ATP hydrolysis

$$
A T P^{4-}+2 \mathrm{H}_{2} \mathrm{O} \rightarrow A D P^{3-}+\mathrm{HPO}_{4}^{2-}+\mathrm{H}_{3} \mathrm{O}^{+} .
$$

and such a soliton travels rather slowly with respect to the speed of longitudinal sound waves. Taking a continuum approximations of the differentialdifference equations results in nonlinear Schrödinger equation(NLS), the solution to which is a soliton. Davydov's work was collaborated by a numerical study(Hyman[21]). They found out that for soliton to form threshold conditions were necessary;

(i) nonlinear cross-coupling between the $C=O$ vibrations and $H . . O$ comprehesion wave must be sufficiently strong and

(ii) the $C=O$ vibrations must be energetic enough to provide a selffocusing effect.

Takeno [35]-[38] proposed an alternative model for propagation of biological energy in the $\alpha$-helix protein. He has argued that the dispersion term in the Davydov model(4.1), may not be appropriate for the migration of vibrational energy. This particular type of exchange interaction is more relevant for electrons or electronic excitons. His approach is basically classical and therefore does not have the constraint of the number of amide-I quanta. He first introduced his Hamiltonian in its simplest form, with acoustic phonons, in order to compare with the Davydov model. Takeno has also generalised his theory to deal with more complex systems where the amide-I energy is coupled to both acoustic and optic phonons.

The the simplified version of Takeno's Hamiltonian is

$$
\begin{gathered}
H_{T}=\sum_{n}\left[\frac{p_{n}^{2}}{2 \mu}+\frac{1}{2} \mu \omega_{0}^{2} \rho_{n}^{2}-2 L \rho_{n+1} \rho_{n}\right] \\
+\sum_{n}\left[\frac{v_{n}^{2}}{2 m}+\frac{1}{2} K_{a}\left(u_{n+1}-u_{n}\right)^{2}\right]+\sum_{n}\left[\frac{1}{2} A_{a} \rho_{n}^{2}\left(u_{n+1}-u_{n-1}\right)\right]
\end{gathered}
$$


Here $\rho_{n}$ and $p_{n}$ are the displacement and momentum coordinates for the high frequency intramolecular (amide-I) oscillator with mass $\mu$ and frequency $\omega_{0}$; L is the coupling strength between neighboring oscillators, which we have restricted to the nearest neighbors. Also, $u_{n}$ and $v_{n}$ are the displacement and momentum coordinates for the molecule at site $\mathrm{n}$; $\mathrm{m}$ and $K_{a}$ are the molecular mass and intramolecular force constant $\left(K_{a}\right.$ is the same as $\mathrm{w}$ in the Davydov model). The last term couples these two oscillating fields with coupling constant $A_{a}$.

In order to make a comparison with the Davydov model, we now for a moment view(4.4) as a quantum Hamiltonian, with the displacement and momentum coordinates replaced by operators. We introduce creation and annihilation operators for the high-frequency oscillator at site $n$ by the equations

$$
\rho_{n}=\sqrt{\frac{\hbar}{2 \mu \omega_{0}}}\left(B_{n}^{\dagger}+B_{n}\right) ; p_{n}=i \sqrt{\frac{\hbar \mu \omega_{0}}{2}}\left(B_{n}^{\dagger}-B_{n}\right)
$$

then the $\rho_{n}$-dependent parts of (4.4) can be written as

$$
\begin{gathered}
H_{v}=\sum_{n} \hbar \omega_{0}\left(B_{n}^{\dagger} B_{n}+\frac{1}{2}\right) \\
-\frac{\hbar L}{\mu \omega_{0}} \sum_{n}\left(B_{n+1}^{\dagger} B_{n}^{\dagger}+B_{n}^{\dagger} B_{n+1}^{\dagger}+B_{n+1}^{\dagger} B_{n}+B_{n+1} B_{n}\right), \\
H^{\prime}=\frac{\hbar A_{a}}{4 \mu \omega_{a}} \sum_{n}\left(B_{n}^{\dagger} B_{n}^{\dagger}+2 B_{n}^{\dagger} B_{n}+B_{n} B_{n}\right)\left(u_{n+1}-u_{n-1}\right) .
\end{gathered}
$$

Comparing(4.7) with the Davydov Hamiltonian it is clear that there are addtional $B_{n}^{\dagger} B_{n}^{\dagger}$ and $B_{n} B_{n}^{\dagger}$ terms both in the dispersive and interaction parts of the quatum version of the Takeno Hamiltonian. The equation operator $B_{n}$ obtained from(4.4) is

$$
\begin{gathered}
i \hbar \dot{B_{n}}=\hbar \omega_{n} B_{n}-\frac{\hbar L}{\mu \omega_{0}}\left(B_{n+1}^{\dagger}+B_{n+1}+B_{n-1}^{\dagger}+B_{n-1}^{\dagger}\right) \\
+\frac{\hbar A_{a}}{2 \mu \omega_{0}}\left(B_{n}^{\dagger}+B_{n}\right)\left(u_{n+1}-u_{n-1}\right) .
\end{gathered}
$$

This differs from equation(4.8), the corresponding equations using Hamiltonian(4.1), by the presence of the creation operators on the righthand side. The presence of those terms means that a phase transformation of the form(4.3) cannot remove the energy of the amide-I quantum $\hbar \omega_{0}=E_{0}$ from the equation. Carrying out that transformation on 
TABLE 1

$\alpha$ helix parameters.

\begin{tabular}{|c|c||c|c|}
\hline Parameter & Value & unit & Reference \\
\hline \hline$E_{0}$ & 1665 & $\mathrm{~cm}^{-1}$ & {$[25]$} \\
\hline $\mathrm{J}$ & 7.8 & $\mathrm{~cm}^{-1}$ & {$[25]$} \\
\hline $\mathrm{w}$ & 13 & $\mathrm{Nm}^{-1}$ & {$[22]$} \\
\hline $\mathrm{m}$ & 114 & $m_{p}$ & {$[30]$} \\
\hline$\chi$ & 0.62 & $10^{-10} m$ & {$[3]$} \\
\hline
\end{tabular}

equation(4.8) produces factors oscillating at $2 \omega_{0}$ in the creation operator terms. In this formulation the magnitude of $E_{0}$ relative to other energies in the problem remains important.

We note that if we drop the creation operators from(4.7), then we can relate the parameters of the two theories by

$$
L=\left(\frac{\mu \omega_{0}}{\hbar}\right) J, A_{a}=\left(\frac{2 \mu \omega_{0}}{\hbar}\right) \chi
$$

The equation of motion derived from classical Hamitonian(4.4) are

$$
\mu \ddot{\rho}_{n}+\mu \omega_{0}^{2} \rho_{n}-2 L\left(\rho_{n+1}+\rho_{n-1}\right)+A_{a} \rho_{n}\left(u_{n+1}-u_{n-1}\right)=0
$$

$$
\left.m \ddot{u}_{n}-K_{a}\left(u_{n+1}-2 u_{n}+u_{n-1}\right)-\frac{1}{2} A_{a}\left(\rho_{n+1}^{2}-\rho_{n-1}\right)^{2}\right)=0 .
$$

Takeno now proceeds by making a continuum approximations to equations (4.10) and (4.11)and obtains this way coupled nonlinear KleinGordon equations for the coordinates $\rho(x, t)$ and $u(x, t)$. A rotating-wave approximation then finally leads to an NLS equation with a classical for the amplitude of the amide-I vibration $\rho(x, t)$ compared to Davydov's NLS equation for the probability amplitude:

$$
i \rho_{t}^{+}+\frac{1}{2 m_{0}} \rho^{+}{ }_{x x}+g|\rho|^{2} \rho^{+}=0,
$$

where $m_{0}=\frac{\omega(k) \mu}{L \gamma l^{2}}$, the nonlinearity parameter is

$$
\begin{gathered}
g=\frac{12 l^{2} A_{a}}{4 m \sqrt{\mu^{2} \omega_{0}^{2}-L \mu+L \mu \gamma l^{2}}\left(c_{2}^{2}-c^{2}\right)} \\
c_{2}=l \sqrt{\frac{K_{a}}{m}} .
\end{gathered}
$$


The self-trapped state of amide-I energy is described by the well-known one-soliton solution

$$
\rho^{+}(x, t)=\alpha \operatorname{sech}\left[\alpha \sqrt{m_{0} g}(x-c t)\right] \exp \left[i\left(k_{1} x-\omega_{1} t\right)\right]
$$

with $k_{1}=m_{0} c, \omega_{1}=\frac{m_{0}}{2} c^{2}-\frac{\alpha^{2}}{2} g$, where $\alpha$ is a constant having the dimension of length. From equation(4.12) the energy $E_{c}$ of solitons is given by $E_{c}=\omega(k)-\left[\frac{\left(k^{2}-k_{1}^{2}\right)}{2 m_{0}}-\frac{\alpha^{2}}{2} g . E_{c}\right.$ becomes

$$
\sqrt{\omega_{0}^{2}-\frac{L}{\mu}}+\frac{k_{1}^{2}}{2 m_{0}}-\frac{\alpha^{2}}{2} g, \text { for } c_{1} k_{1} \ll 1 .
$$

For $c_{1} k_{1} \ll 1$ this is lower than the energy $\omega\left(k_{1}\right)$ of phonon-free vibrons by the factor $\frac{\alpha^{2}}{2} g$ is rferred as the binding energy of vibron soliton. This ensures the stabilty of the vibron solitons as compared with vibrons themselves. The situation described by (4.12) has a finite interval where the amide-I oscillators are excited, accompanied by a lattice displacement which pulls the peptide groups closer together in that region. It is easy to show [it can be done similar the way it was shown by Davydov[14] in the quantum case] that this configuration has a lower energyy than the spatially extended solution to (4.12) and thus self-trapped(self-focusing).

It can be shown that the soliton moving a velocity c carries an energy

$$
E_{c}=E_{0}+\frac{1}{2} m_{t s o l} c^{2}
$$

where $E_{0}$ is the internal energy of the soliton and $m_{t s o l}$ is the effective mass of the soliton.

5. Applying the classical method. It should be noted that although Davydov ,Takeno and others have primary been concerned with the propagation of a soliton in the $\alpha$-helix, the same equations can be used to model electrons travelling along the protein backbone. Cibilis and Cosic[5] took three approaches to examine the possibilty of solitons being linked to the RRM;

(i) Direct comparison with known results on soliton work,

(ii) The derivation of the soliton velocity in terms of fundamental protein parameters based(on Davydov's previous work), and

(iii) An investigation into the bounds of the soliton velocity within a protein, based on the fact that solitons cannot propagate faster than the speed of compression in a protein. 
In this work, we follow the same three above approaches taken by Ciblis and Cosic[5] but here we apply Takeno's work which is a classical approach compared to Davydov work(which is mainly quantun mechanics approach):

(a) Direct comparison with known results on soliton work.

(b) The derivation of the soliton velocity in terms of fundamental protein parameters based(on Takeno's previous work), and

(c) An investigation into the bounds of the soliton velocity within a protein, based on the fact that solitons cannot propagate faster than the speed of compression in a protein.

In Ciblis and Cosic work[5],they used a number of equations presented by Daydov[14], that may be used to define the velocity of a soliton in terms of protein parameters. As mentioned before the equations are based on a one dimensional array of cells coupled via dipole-dipole interactions and a local perturbation of that chain. The equations are as follows;

$$
E_{c}=E_{0}+\frac{1}{2} m_{d s o l} c^{2}
$$

where $E_{0}$ is the internal energy of the soliton, $m_{d s o l}$ is the effective mass of the soliton, $c$ is the velocity of the soliton, $c_{a}$ is the speed of sound through the protein and

$$
m_{d s o l}=\frac{\hbar^{2}}{2 J l^{2}}+\frac{4 \chi^{4}\left(1+\frac{3}{2} s^{2}-\frac{1}{2} s^{4}\right)}{3 w^{2} J c_{a}^{2}\left(1-s^{2}\right)^{3}}
$$

where

$s=\frac{c}{c_{a}}$.

hbar $=$ Plank's constant divided by $2 \pi$

$J=$ dipole-dipole coupling constant between cells of the chain

$l=$ the distance between unit cells

$w=$ the stretching constant

$\omega_{0}=\frac{E_{0}}{\hbar} \chi=$ soliton-phonon coupling constant

Using these equations it is possible to substitute $m_{d s o l}$ into (5.1) and solve the resulting equation for the velocity corresponding to a soliton with energy $E_{c}$ and $E_{0}$. Using Mathematica 3.0 and the result quartic equation provided four possible values of velocity. Ciblis and Cosic varied the values of $E_{0}$ and $E_{c}$ calculated values of a soliton's velocity and thus examined if solitons were implicated in RRM interactions.

In this paper we follow a similar approach used by Cibilis and Cosic, but now we use Takeno's work, and present equations that may define the velocity of a soliton in terms of protein parameters. 


$$
E_{c}=E_{0}+\frac{1}{2} m_{t s o l} c^{2}
$$

Using these equations it is possible to substitute $m_{t s o l}$ into (5.2) and solve the resulting equation for the velocity corresponding to a soliton with energy $E_{c}$ and $E_{0}$. By varying the values of $E_{0}$ and $E_{c}$ it is possible to calculate values of a soliton's velocity and thus examined if solitons were implicated in RRM interactions.

5.1. Direct comparison with known results. A numerical finding (Hyman and others[21]) of solitons in the $\alpha$-helix shows that both solitons and excitons could exist in the $\alpha$-helix of a protein. Their analysis was based on initially approximating a set of difference-differential equations and the initial equations were then decomposed into a coupled system of first order real equations and then solved.They computed the minimum soliton speed near the threshold as $1.26 \times 10^{3} \mathrm{~ms}^{-1}$, which is approximately 0.11 time the speed of a compression wave. The maximum theoretical speed for a soliton was found by Hyman and others to be $1.1 \times 10^{4} \mathrm{~ms}-1$.

The charge velocity approximately computed by RRM estimates is $8 \times 10^{5} \mathrm{~ms}^{-1}$ (see Cosic[11]). Soliton modelled by Hyman and others is slower by several orders of magnitude. It is also greater than the speed of compression for these peripheral amide-I strands.

Cibilis and Cosic[5] suggested that assumptions regarding parameters values may need revision. To investigate this further, a model for calculating the stretching constant, $K_{a}$, for a section of $\alpha$-helix was used to see if the values used in Hyman[21] could be varied. Chou[4] in his modelling work used a value for the stretching constant for a hydrogen bond equal to $13 \mathrm{Nm}^{-1}$. The stretching constant used by Hyman and others was $76 \mathrm{Nm}^{-1}$. Chou[4] provides a method for calculating the stretching constant for a section of $\alpha$-helix. Values for the stretching constant using this method for differing sections of $\alpha$-helix was found to be depedent on the number of constituent of amino acids(see figure1, Cibilis and Cosic[5]). Therefore, $K_{a}$ the stretching constant varies by an order of magnitude of more. This variation in the H-bond constant was also noted by Scott[31]. Thus, a change in the assumed value may have on the soliton velocity predicted, and numerous cases with differing values of (of an order or more) for $K_{a}$ need be considered.

5.2. How does a classical model compare previous results. If our model is applied to predict the velocity of a soliton in the backbone(In particular N-C-C chain). Our model will give better results than the results obtained by Ciblis and Cosic[5] for the following reasons.

Although vibron solitons in the present theory in our model and those in Davydov theory used by Ciblis and Cosic[5] are both described by NLS equation, their natures are fairly different from each other. This stems from 
the difference of model Hamiltonian for amide-I vibrons in helical proteins to which the discussion given better is applicable, provided inter-spine interactions are neglected. Namely, vibrons in the present are described by a set of coupled molecular vibration oscillators as given by the first term of the Takeno's Hamitonian(4.4), where as the corresponding ones in the Davydov's theory are regarded as being of quantal nature having the form of excitons with transfer by exchange interactions. The NLS equation here arises from modulations of vibrons by nonlinear coupling with acoustic phonon propating along the helics of the $\alpha$-proteins, while that in the Davydov theory directly follows from the quantal Schrodinger equation for exicton probability. The present classical picture of vibron solitons appears to be more appropriate to describe vibrational energy transfer in $\alpha$-helical proteins as a mobile entity of conformal change. The same explanation holds for application to backbone chain. Additional studies should be taken in the application of Takeno's Hamiltonian to see if they would cause an electron to propagete through a protein backbone.

\section{REFERENCES}

[1] V. Ya. Antonchenko, A.S. Davydov and A. V. Zolotariuk, Solitons and Proton Motion in ice-like Structures, Phys. Stat. Sol. (b) 115 ,(1983), pp.631-640.

[2] G. Careri, F.Bruni, G. Consolini, Proteins in hydrated protein powders solitons , In Nonlinear Excitation in Biomolecules, M. Peyrard(editor), Les Editions de physique-Spring Herlag, Paris 1996.

[3] G. Careri, U. Buontempo, F. Galluzzi, A.C. Scott, E. Gratton, E. ShymaSUNDER, Spectroscopic evidence for Davydov-like solitons in acetanilide, Phy. Rev. 30B, (1984), pp. 4689-4702.

[4] K. CHOU, Identification of low-frequency modes in protein molecules, Biochem. J., 215(1983), pp.465-469.

[5] P. Ciblis, I. Cosic, The posssibity of soliton/exciton transfer in proteins, J. Theor. Biol. 184, (1997), pp.331-338.

[6] I. Cosic, V. Vojisavljevic, M. Pavlovic, Prediction of 'hot spots' in interlkin2 based on information spectrum charactersitics of growth regulating factors, Biochimie, 71(1989), pp.333-342.

[7] I. Cosic, V. Vojis AVlJevic, M. PAVlovic, The relationship of the resonant recognition model to effects of low intensity light in cell growth, Int. J. Radiat. Biol., 56(1989), pp.179-191.

[8] I. Cosic, Resonant recognition model of protein-protein and protein -DNA interaction, Bioinstrumentation and Biosensors, D.Wise(eds.) Marcel Dekker Inc. New York 1990, pp.475-510.

[9] I. Cosic, Correlation between predicted and measured characteristic frequency of chymotrypsin activation, Proc. 15tg Annu. Conf. IEEE Eng. Med. Biolog. Soc. (1993), pp.1541-1542.

[10] I. CosIC, Macromolecular Bioactivity: is it resonant interaction between macromolecules? - Theory and applications, IEEE Transactions on Biomedical Engineering, 41, (1994), pp.1101-1114.

[11] I. Cosic, Photorecepors having a similar structure but different absorptions can be distinguished using the RRM, IEEE EMBS, 16, (1994), pp.265-266.

[12] T.E Creighton, Proteins, in Chaos and Quantum Physics, M.-J. Giannoni, A. Voros, and J. Zinn-Justin (eds.), 2nd Edition, W.H Freeman, NY, 1993.

[13] A.S. Davydov, The theory of contraction Proteins under their excitation, J. Theor. 
Biol. 38, (1973), pp.559-569.

[14] A.S. Davydov, Biology and quantum mechanics, Pergamon press, New York 1982

[15] D.W Deamer, J.W. Nichols, Proton flux in model biological mebranes, J. Membrane. Biol. 107, (1989), pp.91-103.

[16] R.K. Dodd, J.C. Eilbeck, J.D. Gibbon, H.C. Morris Solitons and nonlinear wave equations, Academic press, London 1982.

[17] R. Drazin, R.S. Johnson Solitons: An introduction, Cambridge University press, NY. 1988.

[18] X. DuAN, S. Scheiner, Modeling of coupled proton transfers by analytic functions, International Journal of quatum chemistry: Quantum Biology Symposium 19, (1992), pp. 109-124.

[19] J. HALding AND P.S. LOMdahl, Two component soliton model for proton transport in hydrogen-bonded molecular chains, Phys. Rev. A 37,(1988), pp.2608-2613.

[20] W.G. Hoover, Canonical dynamics:Equilibruim phase-space distributions, Phys. Rev. A 31,(1985), pp.1695-1697.

[21] J.M. Hyman, D.W. MCLaughlin, A.C. Scott, On Davydov's alpha-helix solitons, Physica D-Nonlinear Phenomena 3, (1981) pp.23-44.

[22] K. Ітон, T. Shimanouchi, Vibrational spectra of crystalline formamide, J. Molec. Spectrosc. 42, (1972) pp.86-99.

[23] V.N. Kharkyanen, E.G. Petrov, I.I. Ukrainskil, Donor-acceptor model of electron transfer through protein, J. Theor. Biol. 73, (1978), pp.29-50.

[24] J.F, NAGLE, M. Mille, H.J. Morowitz, Theory of hydrogen bonded chains in bioenergetics, J. Chem. Phys. 72,(1980), pp.3959-3971.

[25] N.A, Nevskaya, Yu.N, ChiRgadze, Infrared spectra resonance interactions of amide-I and amide-II vibrations in the $\alpha$ helix, Biopolymers 15,(1976), pp.637648.

[26] S. Nose, A unified formulation of the constant temperature molecular dynamics methods Theory of hydrogen, J. Chem. Phys. 81,(1984), pp.511.

[27] E. Nylund, G. Tsironis, Evidence for solitons in hydrogen-bonded systems, Phys. Rev. Lett. 66, (1991), pp.1886-1889.

[28] M. Peyrard, St. Pnevmatikos, N. Flytzanis, Dynamics of the two-component solitary waves in hydrogen-bonded chains, Phys. Rev. A 36, (1987), pp.903-914.

[29] A.V. Savin, A.V. Zolotaryuk, Dynamics of ionic defects and lattice solitons in a thermalized hydrogen-bonded chain, Phys. Rev. A 44, (1991), pp.8167-8183.

[30] A.C. Scotт, Dynamics of Davydov solitons , Phys. Rev. A. 26, (1982), pp.578-595.

[31] A.C. ScotT, Davydov's soliton revisted, Physica D. 51, (1991), pp.333-342.

[32] J. Singh, J.M Thornton, Atlas of protein side-chain interactions, Oxford University Press, NY, 1992.

[33] N.D Sokolov, M.V Vener, V.A. SaVel'Ev, Tentatively study of strong hydrogen bond dynamics: Part II vibrational frequencies considerations, J. of Molecular Structure 222, (1990), pp. 365-386.

[34] W.P. Su, J.R. Scrieffer, A.J. Heeger, Solitons excitations in polycetylene, Phys. Rev. B22,(1980), pp.2099-2111.

[35] S. Takeno, Exciton Soliton in one-dimensional molecular cristals, Prog. theor. Phys. 69,(1983), pp.1798-1801.

[36] S. TAKeno, Vibron soliton in one-dimensional molecular cristals, Prog. theor. Phys. 71,(1984), pp.395-398.

[37] S. TAKENO, Vibron soliton and coherent polarization in an exactly tractible oscillator-lattice system, Prog. theor. Phys. 73,(1985), pp.853-873.

[38] S. TAKENO, Vibron soliton and soliton-induced infrared spectra of crystalline acetanilide, Prog. theor. Phys. 75,(1986), pp.1-14.

[39] G. Tsironis, Proton-solitons bridge physics with biology, In Nonlinear Excitations in Biomolecules, M. Peyrard(editor), Les Editions de Physique-Springer Verlag, Paris 1995.

[40] V. VelJkovic, I. Slavic, General model of pseudopotentials, Phys. Rev. Lett., $29,(1972)$, pp.105-108. 
[41] S. Yomosa, Dynamics of protons in one-dimension hydrogen bonded systems, J. Phys. Soc. Japan, 51, (1982), pp.3318-3324. 